\title{
¿DEPENDE EL DESARROLLO DE LA RAN DE LA EDAD DE INCIO DE LA LECTOESCRITURA?
}

\author{
Roberta Ceccato \\ Dpto. Psicología Evolutiva y de la Educación. Universitat de València. \\ roberta.ceccato@uv.es \\ Lorena Caballero-Gascón \\ Dpto. Psicología Básica, Clínica y Psicobiología. Universitat Jaume I, \\ Olga Fernandez-García \\ Dpto. Psicología Evolutiva y de la Educación. Universitat de València. \\ Isabel Chaves \\ Dpto. Psicología Evolutiva y de la Educación. Universitat de València. \\ Ma Dolores Gil-Llario \\ Dpto. Psicología Evolutiva y de la Educación. Universitat de València.
}

\section{RESUMEN}

Antecedentes: La habilidad del alumno para nombrar rápidamente estímulos familiares (RAN) es un factor implicado en el rendimiento lector evaluable de forma indiscriminada a lo largo de los primeros años de escolarización. El objetivo de este estudio, pues, es analizar si la adquisición de dicha habilidad depende de variables relacionadas con una iniciación temprana a la lecto-escritura o si su desarrollo se relaciona con factores de madurez cronológica. Método: Para ello se aplicó la prueba RAN a 71 niños de 6-7 años croatas (cultura en la que la iniciación a la lectura es más tardía); a 44 niños de 4-5 años españoles (equiparando los dos grupos por edad de inicio de la lecto-escritura) y a 43 niños españoles de 6-7 años (equiparando los dos grupos por edad cronológica). Los resultados muestran que comparando el grupo de niños croatas con el grupo de niños españoles de 45 años, se destacan diferencias significativas en los tiempos de nombramiento de los estímulos de los 4 diferentes componentes de la prueba. Los niños mayores reconocen más rápidamente los números y letras mientras que los más pequeños tardan más en estos estímulos reconociendo más rápidamente los objetos y colores. Sin embargo, comparando los dos grupos de niños de 6-7 años (los croatas y los españoles) no se han encontrado diferencias estadísticamente significativas. Se concluye pues, que el desarrollo de la RAN se caracteriza por una adquisición más temprana de los estímulos no simbólicos (objetos-colores) que de los estímulos simbólicos (letras-números) y que dicha adquisición se relaciona más con factores de madurez cronológica que de edad de inicio de la 
lecto-escritura. Esta habilidad, por lo tanto, a pesar de estar estrechamente relacionada con el aprendizaje de la lectura y de la escritura, parece desarrollarse de forma independiente a la edad de inicio de dicho aprendizaje.

Palabras clave: RAN; lectoescritura; aprendizaje lector; velocidad de nombramiento

\section{ABSTRACT \\ Does the development of ran depend on the age of the beginning of reading learning process?}

Background: The ability of the student to quickly name familiar stimuli (RAN) is a factor involved in reading performance that can be evaluated indiscriminately throughout the first years of schooling. The objective of this study, then, is to analyze if the acquisition of this ability depends on variables related to an early initiation to the reading-writing or if its development is related to factors of chronological maturity. Method: To this end, the RAN test was applied to 71 6-7 years old children from Croatia (culture in which the initiation to reading is later); to 44 4-5 years old Spanish children (equating the two groups by age of beginning of the reading-writing) and to 43 6-7 years old Spanish children (equating the two groups by chronological age). The results show that by comparing the group of Croatian children with the group of Spanish children aged 4-5 years, significant differences stand out in the appointment times of the stimuli of the 4 different components of the test. Older children recognize numbers and letters more quickly while younger ones take longer in these stimuli, recognizing objects and colors more quickly. However, comparing the two groups of children aged 6-7 years (Croatians and Spanish), no statistically significant differences were found. It is concluded that the development of the RAN is characterized by an earlier acquisition of nonsymbolic stimuli (objects-colors) than symbolic stimuli (letters-numbers) and that this acquisition is related more to factors of chronological maturity than of age of beginning of the reading-writing. This ability, therefore, despite being closely related to the learning of reading and writing, seems to develop independently of the age at which such learning begins.

Keywords: RAN; reading and writing; learning; rapid naming

\section{ANTECEDENTES:}

Una habilidad cognitiva básica que frecuentemente se ha asociado a la habilidad lectora es el reconocimiento rápido y automático de items visuales (Kirby, Roth, Desrochers \& Lai, 2008).

Dicha decodificación rápida (Rapid Automatized Naming, en adelante RAN) hace referencia al tiempo que emplea un niño para nombrar rápidamente y con precisión una serie de estímulos visuales familiares (generalmente letras, dígitos, objetos y colores). Norton y Wolf (2011) recientemente sostuvieron que la RAN constituye "un microcosmos o mini-circuito de un circuito de lectoescritura, cuyo desarrollo es posterior", apelando al hecho que ambos sistemas implican procesos cognitivos estrechamente relacionados. Efectivamente, tanto las tareas de la RAN como el proceso lectoescritor implican: atención al estímulo; procesos visuales responsables de la detección inicial de las características del estímulo; decodificación y conversión grafema-fonema; integración de la información visual con la ortográfica y las representaciones fonológicas almacenadas en la memoria; procesos léxicos como el acceso y la recuperación de los códigos fonológicos; y organización de la producción articulatoria (Araújo, Petersson, Reis \& Faísca, 2014).

Un grupo sustancial de investigaciones identifica la RAN como uno de los mejores (y quizás uno de los más universales, longitudinales y actuales) predictores de la capacidad lectoescritora y también de la detección de un posible déficit en el desarrollo de la misma (Araújo, Inácio, Francisco, Faísca, Petersson \& Reis, 2011; Kirby, Parrila \& Pfeiffer, 2003; Kirby et al., 2008; Vaessen, Bertrand, Tóth, Csépe, Faísca, Reis \& Blomert, 2010; Wolf, Bowers \& Biddle, 2000; Wolf, O’Rourke, Gidney, 
Lovett, Cirino \& Morris, 2002). Sin embargo, en la literatura existe un gran desacuerdo por lo que se refiere a la intensidad de la relación entre RAN y lectoescritura. Algunos estudios refieren la existencia de una estrecha correlación entre las mediciones de la velocidad de nombramiento y las de rendimiento lectoescritor (Babayig it \& Stainthorp, 2010), otros subrayan una relación débil (Cunningham, 2006) y finalmente algunos destacan una relación casi insignificante (Scaborough, 1998).

Así mismo, muchas son las teorías que analizan e intentan definir la relación entre RAN y lectoescritura y, actualmente, no hay un consenso común con respecto a cuáles son los mecanismos responsables de dicha relación. Mientras algunos autores sugieren que la RAN refleja principalmente el acceso y la recuperación en la memoria a largo plazo de los códigos fonológicos (Chiappe, Stringer, Siegel \& Stanovich, 2002; Pennington, Cardoso-Martins, Green \& Lefly, 2001; Schatschneider, Carlson, Francis, Foorman \& Fletcher, 2002), otros apoyan la hipótesis de que la RAN constituye un proceso lectoescritor "no-fonológico" independiente (Wolf \& Bowers, 1999).

Así mismo, muchos estudios han demostrado que la RAN presenta una relación más fuerte con tareas de lectoescritura que están basadas en el reconocimiento y reproducción escrita de palabras que presentan patrones ortográficos específicos (como por ejemplo las palabras irregulares) con respecto a las tareas de lectura o escritura que requieren una decodificación fonológica, como lo son el reconocimiento y la reproducción escrita de pseudopalabras (Araújo et al., 2011; Clarke, Hulme \& Snowling, 2005; Georgiou, Parrilla, Kirby \& Stephenson, 2008; Savage et al., 2005). En estos resultados se apoya la hipótesis que plantea que la RAN refleje la involucración de procesos de decodificación ortográfica, aunque la exacta naturaleza de dichos procesos aún no queda clara. Por ejemplo, Manis, Seidenberg \& Doi (1999), quienes se centraron principalmente en los procesos ortográficos, sugirieron que uno de los aspectos cruciales de la RAN consiste en la atribución arbitraria de los sonidos a los estímulos que componen las tareas. Sin embargo, Bowers y colaboradores (Bowers \& Wolf, 1993; Bowers \& Newby-Clark, 2002) propusieron que una lenta velocidad de procesamiento destacada en las tareas de la RAN impide el proceso de integración de la información visual de la secuencia de letras que componen una palabra, lo cual es necesario para la identificación de los patrones ortográficos y, consecuentemente, para la formación de un léxico ortográfico que permita al niño una lectura y escritura fluida y rápida. No obstante, otros estudios subrayaron que la RAN se relaciona de forma similar tanto con la lectoescritura de palabras como de pseudopalabras (Moll et al., 2009; Savage, Pillay \& Melidona, 2007), Lo cual es difícil de conciliar con una explicación puramente ortográfica.

Por otro lado, en un metanálisis, Swanson, Trainin, Necoechea y Hammill (2003) analizaron la relación existente entre conciencia fonológica, RAN y desarrollo lectoescritor y encontraron que la conciencia fonológica y la RAN se relacionaban de forma moderada con la lectoescritura de palabras. De forma similar, Ziegler y colaboradores (2010) demostraron que, controlando estadísticamente la conciencia fonológica, la asociación entre RAN y lectura y escritura de palabras era débil y limitada simplemente a la fluidez de dicha lectoescritura, pero no a la exactitud de la misma. Por lo tanto, los autores concluyeron que es el componente fonológico que la RAN comparte con la conciencia fonológica lo que hace de la RAN un importante indicador de la habilidad de lectoescritura.

Además de las diferentes teorías propuestas para explicar la naturaleza de la relación entre RAN y lectoescritura, existen otras muchas variables que concurren en esta falta de consenso sobre la RAN y su rol en el inicio de la lectoescritura. Entre ellas, la falta de aclaración sobre la el origen de la importancia de la RAN y sobre cuáles serían las variables que desencadenan y apoyan su desarrollo.

Así pues, el objetivo de este trabajo es analizar si la adquisición de dicha habilidad depende de 
variables relacionadas con una iniciación temprana a la lecto-escritura o si su desarrollo se relaciona con factores de madurez cronológica.

\section{MUESTRA 0 PARTICIPANTES:}

Los participantes a este estudio fueron 71 niños de 6-7 años croatas (cultura en la que la iniciación a la lectura es más tardía); 44 niños de 4-5 años españoles (con la finalidad de equiparar el grupo español y el croata por edad de inicio de la lecto-escritura) y 43 niños españoles de 6-7 años (con la finalidad de equiparar el grupo español y el croata por edad cronológica). Como se destaca en los gráficos 1, 2 y 3 , en el grupo de niños croatas de 6-7 años, el 60\% eran niñas y el $40 \%$ niños; en el grupo de participantes españoles de $4-5$ años el $55 \%$ eran niñas y el $45 \%$ niños; mientras que el en grupo de niños españoles de 6-7 años, el 51\% eran chicas y el $49 \%$ chicos.

Gráfico 1: Participantes croatas de 6-7 años

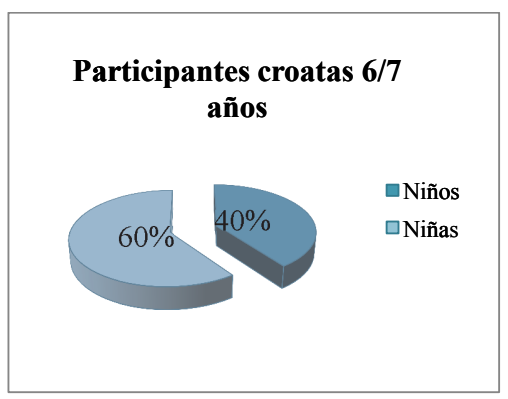

Gráfico 2: Participantes españoles de 4-5 años

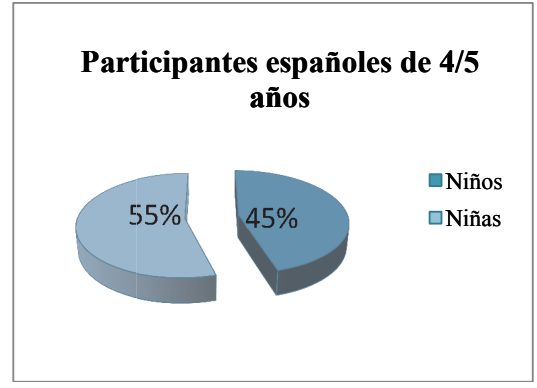

Gráfico 3: Participantes españoles de 6-7 años

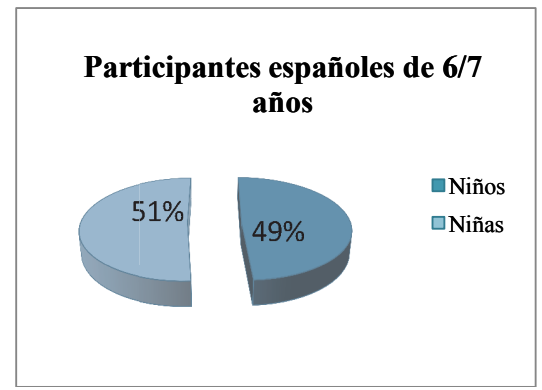




\section{METODOLOGIA E INSTRUMENTOS UTILIZADOS:}

A cada grupo de participantes de administró de forma individual la prueba "The Rapid Automatized Naming Test" (RAN; Wolf y Denkla, 2003). La tarea consiste en nombrar lo más rápidamente posible 200 estímulos de 4 tipos diferentes (números, letras, colores e imágenes) agrupados en cuatro subtests: RAN-números, RAN-letras, RAN-colores y RAN-imágenes. Los 50 estímuIos de cada subtest se presentan en diferentes cartulinas de $21 \times 30 \mathrm{~cm}$ organizados en 5 filas de 10 estímulos cada una.

En cada subtest los estímulos son presentados aleatoriamente pero siempre 50 de cada uno de ellos. En la hoja de registro de la RAN se anota el tiempo que tarda el/la alumno/a en nombrar todos los estímulos de cada cartulina y el número de errores que comete al nombrarlos. Con estos datos se obtiene un índice de eficiencia de cada subtest según el procedimiento descrito por Compton (2003) que consiste en convertir las puntuaciones en números/segundo, letras/segundo, colores/segundo e imágenes/segundo. La fiabilidad medida con el Alpha de Cronbach es de .809.

\section{RESULTADOS:}

En primer lugar, se compararon los tiempos de respuesta en los dos grupos equiparados por el momento de inicio de la lecto-escritura, es decir el grupo de niños croatas de 6/7 años y el grupo de niños españoles de $4 / 5$ años.

Como se destaca en los gráficos 4 y 5 , existen diferencias significativas en los tiempos de nombramiento de los estímulos de los 4 diferentes componentes de la prueba. Los niños mayores (es decir, los niños croatas) reconocen más rápidamente los números y letras mientras que los más pequeños (los españoles) tardan más en estos estímulos reconociendo más rápidamente los objetos y colores.

Gráfico 4: Velocidad de nombramiento en niños croatas de 6-7 años.

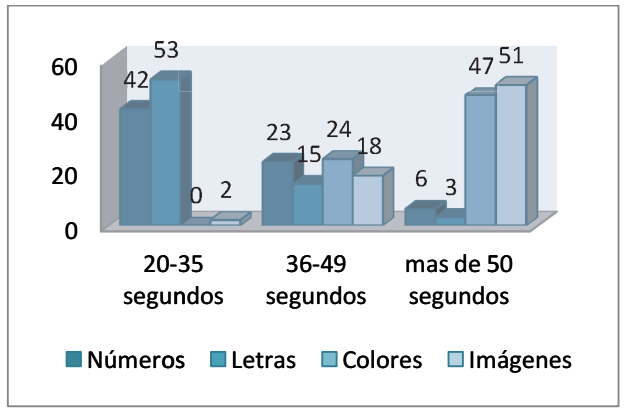




\section{¿DEPENDE EL DESARROLLO DE LA RAN DE LA EDAD DE INCIO DE LA LECTOESCRITURA?}

Gráfico 5: Velocidad de nombramiento en niños españoles de 4-5 años.

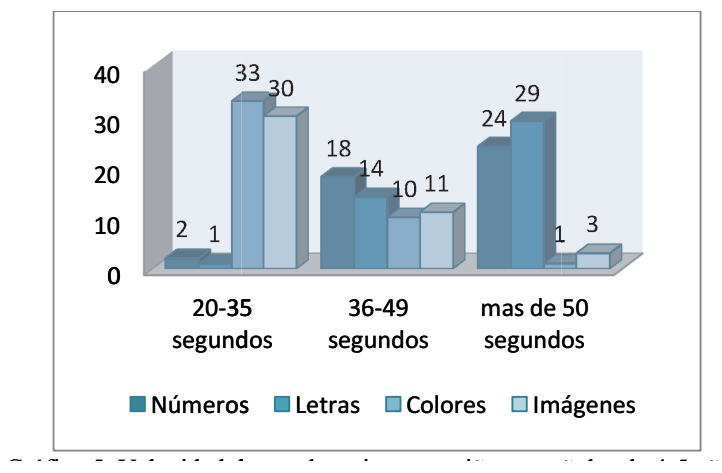

A continuación, se compararon los grupos equiparados por edad cronológica, es decir el grupo de niños croatas de 6-7 años y el grupo de niños españoles de la misma edad.

Como se destaca en los gráficos 6 y 7 , al comparar los tiempos de nombramiento de estos dos grupos, no se han encontrado diferencias significativas, ya que ambos parecen reconocer y nombrar de forma más automática y veloz los estímulos arbitrarios de números y letras que los estímulos más naturales como colores e imágenes.

Gráfico 6: Velocidad de nombramiento en niños croatas de 6-7 años.

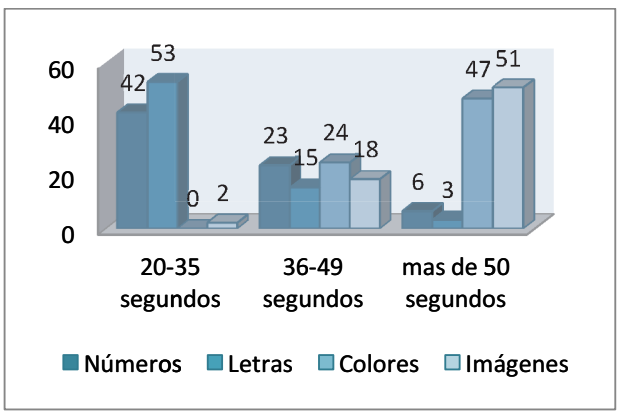

Gráfico 7: Velocidad de nombramiento en niños españoles de 6-7 años.

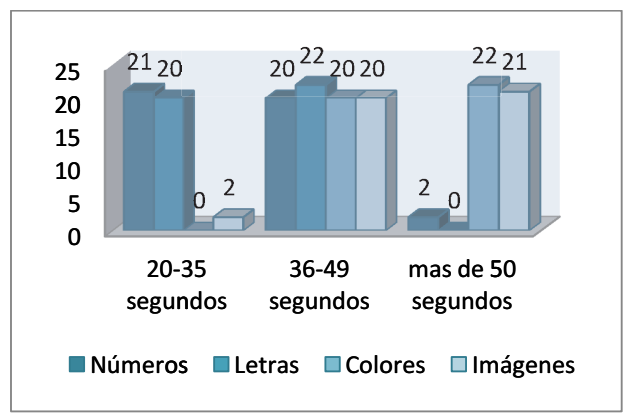




\section{DISCUSIÓN Y CONCLUSIONES:}

Los resultados encontrados nos permiten afirmar que hay una gran diferencia entre el desarroIlo de la RAN en niños más pequeños de 4-5 años y en chicos más mayores de 6-7 años. Los primeros, efectivamente, parecen reconocer de forma más automática estímulos naturales cuyo aprendizaje no depende de la escolarización y la alfabetización adquirida, como lo son los colores y las imágenes. Sin embargo, los niños más mayores parecen reconocer y nombrar de forma más rápida aquellos estímulos cuya asociación grafema-fonema se debe a una asignación totalmente arbitraria y cuyo aprendizaje se debe al proceso de escolarización y enseñanza de la lectoescritura.

Efectivamente, al comparar los dos grupos equiparados por edad cronológica (niños españoles y croatas de 6-7 años), no se han encontrado diferencias entre los tiempos de nombramiento de los diferentes estímulos que componen la prueba RAN. Este dato, sin embargo, no deja de llamar la atención ya que, a diferencia de los niños españoles de 6-7 años, los niños croatas que han participado en nuestro estudio acaban de iniciarse a la lectoescritura, por lo que exactamente estímulos relacionados con un aprendizaje arbitrario deberían ser los que menos fácilmente se reconocen.

Nuestros resultados, pues, parecen ir en la misma línea de aquellos investigadores que apoyan la hipótesis que plantea que la RAN refleje la involucración de procesos de decodificación ortográfica. (Araújo et al., 2011; Clarke, Hulme \& Snowling, 2005; Georgiou, Parrilla, Kirby \& Stephenson, 2008; Savage et al., 2005). Así mismo, se confirman las afirmaciones de Manis, Seidenberg \& Doi (1999), quienes, centrándose principalmente en los procesos ortográficos, sugirieron que uno de los aspectos cruciales de la RAN consiste en la atribución arbitraria de los sonidos a los estímulos que componen las tareas.

A la luz de lo anteriormente expuesto y de los datos presentes en la literatura, nos parece razonable afirmar y concluir que el desarrollo de la RAN se caracteriza por una adquisición más temprana de los estímulos no simbólicos (objetos-colores) que de los estímulos simbólicos (letrasnúmeros) y que dicha adquisición se relaciona más con factores de madurez cronológica que de edad de inicio de la lecto-escritura. Esta habilidad, por lo tanto, a pesar de estar estrechamente relacionada con el aprendizaje de la lectura y de la escritura, parece desarrollarse de forma independiente a la edad de inicio de dicho aprendizaje.

\section{REFERENCIAS BIBLIOGRÁFICAS:}

Araújo, S., Inácio, F., Francisco, A., Faísca, L., Petersson, K. M., \& Reis, A. (2011). Component processes subserving rapid automatized naming in dyslexic and non-dyslexic readers. Dyslexia, 17, 242-255. doi: 10.1002/dys.433

Araújo, S., Reis, A., Petersson, K. M., \& Faísca, L. (2014, October 20). Rapid Automatized Naming and Reading Performance: A Meta-Analysis. Journal of Educational Psychology, 2, 1-16.

Cooper, H. M. (2010). Research synthesis and meta-analysis: A step-bystep approach (4th ed.). Thousand Oaks, CA: Sage.

Cunningham, A. E. (2006). Accounting for children's orthographic learning while reading text: Do children self-teach? Journal of Experimental Child Psychology, 95, 56-77.

Cutting, L. E., \& Denckla, M. B. (2001). The relationship of serial rapid naming and word reading in normally developing readers: An exploratory model. Reading and Writing, 14, 673-705. doi:10.1023/A:1012047622541

Kirby, J. R., Parrila, R. K., \& Pfeiffer, S. L. (2003). Naming speed and phonological awareness as predictors of reading development. Journal of Educational Psychology, 95, 453-464. doi:10.1037/0022-0663.95.3.453

Kirby, J. R., Roth, L., Desrochers, A., \& Lai, S. (2008). Longitudinal predictors of word reading development. Canadian Psychology, 49, 103-110. doi:10.1037/07085591.49.2.103 
Manis, F. R., Seidenberg, M. S., \& Doi, L. M. (1999). See Dick RAN: Rapid naming and the logitudinal prediction of reading subskills in first and second graders. Scientific Studies of Reading, 3, 129-157. doi: 10.1207/s1532799xssr0302_3

Norton, E. S., \& Wolf, M. (2011). Rapid automatized naming (RAN) and reading fluency: Implications for understanding and treatment of reading disabilities. Annual Review of Psychology, 63, 427-452.

Scarborough, H. S. (1998). Predicting the future achievement of second graders with reading disabilities: Contributions of phonemic awareness, verbal memory, rapid naming, and IQ. Annals of Dyslexia, 48, 115-136. doi:10.1007/s11881-998-0006-5

Schatschneider, C., Carlson, C. D., Francis, D. J., Foorman, B. R., \& Fletcher, J. M. (2002). Relationship of rapid automatized naming and phonological awareness in early reading development: Implications for the double-deficit hypothesis. Journal of Learning Disabilities, 35, 245-256. doi:10.1177/002221940203500306

Schatschneider, C., Fletcher, J. M., Francis, D. J., Carlson, C. D., \& Foorman, B. R. (2004). Kindergarten prediction of reading skills: A longitudinal comparative analysis. Journal of Educational Psychology, 96, 265-282. doi:10.1037/0022-0663.96.2.265

Vaessen, A., \& Blomert, L. (2010). Long-term cognitive dynamics of fluent reading development. Journal of Experimental Child Psychology, 105, 213-231. doi:10.1016/j.jecp.2009.11.005

Vaessen, A., Bertrand, D., Tóth, D., Csépe, V., Faísca, L., Reis, A., \& Blomert, L. (2010). Cognitive development of fluent word reading does not qualitatively differ between transparent and opaque orthographies. Journal of Educational Psychology, 102, 827- 842. doi:10.1037/a0019465

Vaessen, A., Gerretsen, P., \& Blomert, L. (2009). Naming problems do not reflect a second independent core deficit in dyslexia: Double déficits explored. Journal of Experimental Child Psychology, 103, 202-221. doi:10.1016/j.jecp.2008.12.004

Wolf, M., \& Bowers, P. G. (1999). The double-deficit hypothesis for the developmental dyslexias. Journal of Educational Psychology, 91, 415-438. doi:10.1037/0022-0663.91.3.415

Wolf, M., Bowers, P. G., \& Biddle, K. (2000). Naming-speed processes, timing, and reading: A conceptual review. Journal of Learning Disabilities, 33, 387-407. doi:10.1177/002221940003300409

Wolf, M., O'Rourke, A. G., Gidney, C., Lovett, M., Cirino, P., \& Morris, R. (2002). The second deficit: An investigation of the independence of phonological and naming speed deficits in developmental dyslexia. Reading and Writing, 15, 43-72. doi:10.1023/A:1013816320290 